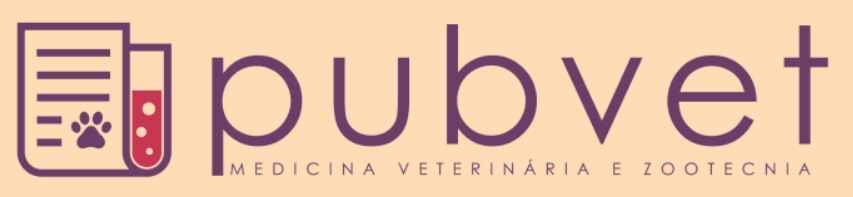

https://doi.org/10.22256/pubvet.v12n6a102.1-6

\title{
Leptospirose canina: Relato de caso
}

\author{
Raquel Albuquerque Silva ${ }^{\bullet}{ }^{1 *}$, Marcelo Campos Rodrigues ${ }^{\bullet}$, Misael das Virgens \\ Santana $^{\ominus}$, Karoline Figueredo Rodrigues $\odot$ 1, Fernando Barbosa de Sousa ${ }^{\oplus}$, Thiago \\ Sousa da Silva ${ }^{\bullet}$, Kellen Matuzzy Silva de Melo ${ }^{\bullet} 1$
}

${ }^{1}$ Médico(a) Veterinário(a), Residente em clínica e cirurgia de cães e gatos, Universidade Federal do Piauí (UFPI), Teresina, Piauí, Brasil. ${ }^{2}$ Médico Veterinário, Doutor em Ciência Veterinária pela Universidade Federal Rural de Pernambuco (UFRPE), professor de Clinica Cirúrgica Veterinária da Universidade Federal do Piauí (UFPI), Teresina, Piauí, Brasil.

${ }^{3}$ Médico Veterinário, Residente em Reprodução Animal, Universidade Federal do Piauí (UFPI). Teresina, Piauí, Brasil. *Autor para correspondência por e-mail: raquel_albuq@hotmail.com

RESUMO. A Leptospirose é uma zoonose de distribuição mundial que apresenta o cão como importante hospedeiro, sendo crucial seu diagnóstico e tratamento nesta espécie, que possui estreita relação com o homem. Um canino foi atendido no Hospital Veterinário da Universidade Federal do Piauí em Teresina, Piauí, apresentando histórico de hematúria, hiporexia e letargia há dois dias. O exame físico revelou letargia, mucosas oral, ocular e peniana ictéricas, dor à palpação abdominal e febre. Na análise hematológica foi detectada anemia macrocítica hipocrômica, trombocitopenia, leucocitose por neutrofilia, além de um aumento nos valores de ureia, AST, ALT, fosfatase alcalina e bilirrubinas. O exame de soroaglutinação microscópica mostrou resultado reagente a Leptospira interrogans sorovar Australis na titulação 1:100. O animal foi efetivamente tratado com Ampicilina, Doxiciclina e terapia de suporte.

Palavras chaves: Australis, canino, Leptospirose

\section{Canine leptospirosis: Case report}

ABSTRACT. Leptospirosis is a zoonosis of worldwide distribution that has the dog as an important host, showing the importance of diagnosing and treating this animal that has a straight relationship with humans. A dog was attended at the Veterinary Hospital of the Federal University of Piauí in Teresina, Piauí, was present with history of hematuria, hyporexia and lethargy. Physical examination revealed lethargy, oral, ocular and penile icteric mucosa, pain at abdominal palpation and pyrexia. Hematologic analysis revealed hypochromic macrocytic anemia, thrombocytopenia, leukocytosis due to neutrophilia, and an increase in urea, AST, ALT, alkaline phosphatase and bilirubin values. The microscopic serum agglutination test showed a reactive result of Leptospira interrogans serovar Australis at the 1: 100 titre. The animal was effectively treated with Ampicillin, Doxycycline and supportive therapy.

Key words: Australis, Canine, Leptospirosis

\section{Leptospirosis canina: Reporte de un caso}

RESUMEN. La leptospirosis es una zoonosis de distribución mundial que presenta al perro como importante huésped, siendo crucial su diagnóstico y tratamiento en esta especie, que tiene estrecha relación con el hombre. Un canino fue atendido en el Hospital Veterinario de la Universidad Federal de Piauí en Teresina, Piauí, presentando histórico de hematuria, hiporexia y letargo desde dos días anteriores. El examen físico reveló letargo, mucosas oral, 
ocular y peneana ictérica, dolor a la palpación abdominal y fiebre. En el análisis hematológico se detectó anemia macrocítica hipocrómica, trombocitopenia, leucocitosis por neutrofilia, además de un aumento en los valores de urea, AST, ALT, fosfatasa alcalina y bilirrubinas. El examen de sueroaglutinación microscópica mostró resultados reactivos a Leptospira interrogans serovar Australis en la titulación 1:100. El animal fue efectivamente tratado con Ampicilina, Doxiciclina y terapia de soporte.

Palabras claves: Australis, Canino, Leptospirosis

\section{Introdução}

A Leptospirose é uma zoonose de distribuição mundial que afeta diversas espécies animais. É causada por uma bactéria espiroqueta patogênica da família Leptospiraceae, gênero Leptospira, da espécie Leptospira interrogans. Mais de 250 sorovares de L. interrogans já foram descritos em diversos países, dentre eles o $L$. icterohaemorrhagiae, L. copenhageni, $L$. grippotyphosa, $L$. australis e L. pomona, $L$. autumnalis e L. sejroe são os mais encontrados em cães (Knöpfler et al. 2017; Schuller 2017).

Após entrarem em contato com o hospedeiro pelas mucosas nasais, orais ou conjuntivais, da pele lesada ou após contato prolongado com a água, as leptospiras rapidamente promovem infecção sistêmica através da corrente sanguínea, atingindo e replicando-se em diversos tecidos, incluindo rim, baço, fígado, sistema nervoso central, olhos e trato genital. Esta fase inicial, denominada leptospiremia, pode persistir por até 10 dias, até o hospedeiro montar uma resposta imunológica efetiva contra a bactéria, eliminando o organismo da corrente sanguínea e da maioria dos tecidos, persistindo somente em locais imunoprivilegiados, como os olhos e os túbulos renais, dando início à fase de leptospiúria, quando o animal elimina as leptospiras no ambiente, via urina (Greene et al. 1993; Hagiwara et al. 2015; $\underline{\text { Schuller 2017). }}$.

Os sinais clínicos dependem da resposta imunológica do hospedeiro frente à infecção e do sorovar infectante. Frequentemente, na fase aguda, são observados sinais de anorexia, vômitos, letargia, febre, dor abdominal, dispneia, taquipneia, icterícia, oligúria, anúria e uveíte (Greene et al. 1993; Hagiwara et al. 2015; Nelson \& Couto 2015; Schuller 2017). O diagnóstico é feito por meio de exames sorológicos, microbiológicos e moleculares, além da anamnese, sinais clínicos e exames hematológicos do paciente. O teste de soroaglutinação microscópica (SAM) é o exame padrão recomendado pela Organização Mundial de Saúde para o diagnóstico confirmatório da doença e consiste na detecção de anticorpos aglutinantes, a partir da reação de diluições do soro do animal com leptospiras vivas (Hagiwara et al. 2015).

$\mathrm{O}$ tratamento consiste em antibioticoterapia e terapia de suporte para os diferentes sistemas acometidos do organismo. As leptospiras são sensíveis a uma ampla variedade de antibióticos, dentre eles a penicilina, ou derivados, e a doxiciclina (usada para eliminar as leptospiras dos túbulos renais). Desta forma, inicia-se o tratamento via intravenosa com penicilina ou derivados (penicilina G, ampicilina ou amoxicilina) para tratar a leptospiremia e finaliza-se com a doxiciclina para tratar a leptospiúria (Schuller et al. 2015; Schuller 2017).

O presente relato tem por objetivo descrever o caso de um canino acometido pela Leptospirose, atendido no Hospital Veterinário da Universidade Federal do Piauí (HVU-UFPI), destacando a importância de diagnosticar a enfermidade e tratar esta espécie, que possui estreita relação com o homem.

\section{Relato de caso}

Foi atendido no HVU-UFPI, Teresina, Piauí (PI), um canino macho, sem raça definida (SRD), de um ano e seis meses de idade, com 16,5 kg de massa corporal. O tutor observou que há um dia o animal apresentava hematúria e há dois dias vinha com letargia e hiporexia. Informou, ainda, que o mesmo só era vacinado contra raiva, convivia com quatro gatos aparentemente saudáveis e não tinha acesso à rua, no entanto, quando chovia, a água dos esgotos escoava para o local onde o cão se localizava.

Ao exame físico foi constatada letargia, aumento de linfonodos submandibulares e poplíteos, mucosas oral, ocular e peniana ictéricas (Figura 1), dor à palpação abdominal, frequência cardíaca de 100 batimentos por minuto, frequência respiratória de 40 movimentos por minuto, temperatura retal de $39,8{ }^{\circ} \mathrm{C}$ e tempo de preenchimento capilar (TPC) de 2 segundos. 
Foram realizados hemograma, perfil bioquímico (ureia, creatinina, alanina aminotransferase [ALT], aspartato aminotransferase $[\mathrm{AST}]$, fosfatase alcalina $[\mathrm{FA}]$, proteínas totais, albumina, globulina, bilirrubina total, bilirrubina conjugada e não conjugada), sumário de urina, soro-aglutinação microscópica e cultura de urina. $\mathrm{Na}$ avaliação sanguínea o animal apresentou anemia macrocítica hipocrômica, trombocitopenia, leucocitose por neutrofilia (Quadro 1), além de um aumento nos valores de uréia, AST, ALT e FA (Quadro 2). No exame físico da urina foi observado coloração escura, densidade elevada e aspecto turvo. No exame químico, detectou-se bilirrubinúria, $\mathrm{pH}$ levemente diminuído e presença de sangue oculto e, na análise do sedimento, pôde-se verificar células epiteliais descamativas e transicionais, cilindros tipo granular grosseiro, duas cruzes de bactérias, cristais de fosfato triplo e bilirrubina.

$\mathrm{O}$ animal foi encaminhado à internação, onde foi dado início ao tratamento com complexo vitamínico $(0,2 \mathrm{ml} / \mathrm{kg}$, IV, SID), solução de cloreto de sódio $0,9 \%(3 \mathrm{ml} / \mathrm{kg} / \mathrm{h}, \mathrm{IV})$, ranitidina $(2 \mathrm{mg} / \mathrm{kg}, \mathrm{SC}, \mathrm{BID})$, penicilina benzantina $(40.000$ UI/ $\mathrm{kg} \mathrm{IM}$, d.u) e dipirona injetável ( $25 \mathrm{mg} / \mathrm{kg}$,IV, TID). Após três dias de internação, foi confirmado o diagnóstico de leptospirose, a partir do exame de soro aglutinação microscópica (MAT), que mostrou resultado reagente ao sorovar australis na titulação 1:100. Desta forma, foi dado início à anti-bioticoterapia com ampicilina ( $25 \mathrm{mg} / \mathrm{kg}$, IV, QID). Nesta ocasião, foi realizado novo hemograma e perfil bioquímico para reavaliação do animal. Em relação ao hemograma anterior, houve aumento do número de leucócitos, aumento do número de plaquetas e persistência da anemia (Quadro 1). No exame bioquímico foi dosada creatinina e as bilirrubinas que não foi possível serem dosadas no exame anterior, apresentando resultado normal e aumentado, respectivamente (Quadro 2). Devido ao quadro de anemia observada nos hemogramas, o animal foi submetido à transfusão sanguínea, utilizando-se a dexametasona ( $1 \mathrm{mg} / \mathrm{kg}$, IV), 10 minutos antes do início do procedimento.

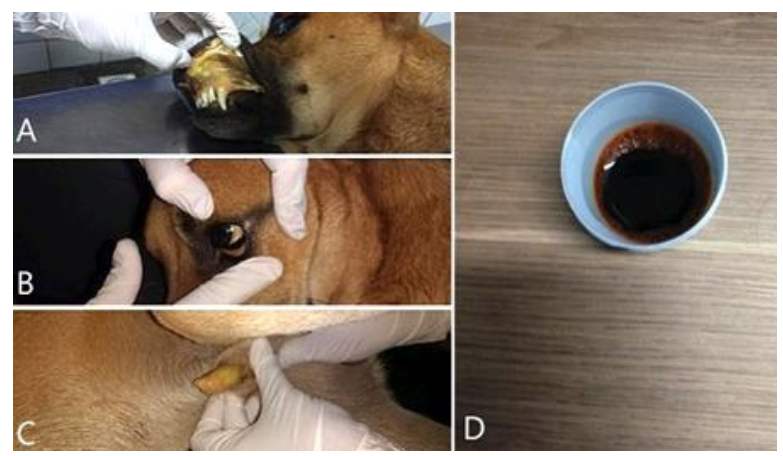

Figura 1. Mucosas de um canino apresentando icterícia e característica macroscópica de sua urina. A- Mucosa oral; B - Mucosa conjuntival; C - Mucosa peniana; D - Aspecto macroscópico da urina.

Quadro 1. Resultados de hemogramas de um cão com leptospirose, nos dias 0, 3 e 8 de tratamento, atendido no HVU/UFPI, Teresina-PI em 2017.

\begin{tabular}{|c|c|c|c|c|c|c|}
\hline Eritrograma & \multicolumn{2}{|c|}{ Resultado Dia 0} & \multicolumn{2}{|c|}{ Resultado Dia 3} & \multicolumn{2}{|c|}{ Resultado Dia 8} \\
\hline $\begin{array}{l}\text { Hemácias }\left(\times 10^{6} / \mu \mathrm{L}\right) \\
\text { Hemoglobina }(\mathrm{g} / \mathrm{dL}) \\
\text { Hematócrito }(\%) \\
\text { VCM (fL) } \\
\text { CHCM }(\%) \\
\text { Plaquetas }\left(\times 10^{3} / \mu \mathrm{L}\right)\end{array}$ & & & & & & \\
\hline \multirow[t]{2}{*}{ Leucograma } & \multicolumn{2}{|c|}{ Resultado dia 0} & \multicolumn{2}{|c|}{ Resultado dia 3} & \multicolumn{2}{|c|}{ Resultado dia 8} \\
\hline & REL \% & ABS $/ \square 1$ & REL \% & ABS $/ \square 1$ & REL \% & $\mathbf{A B S} / \square 1$ \\
\hline Leucócitos totais & - & 32.600 & - & 40.700 & - & 15.620 \\
\hline Mielócitos & 0 & 0 & 0 & 0 & 0 & 0 \\
\hline Metamielócitos & 0 & 0 & 0 & 0 & 0 & 0 \\
\hline Bastonetes & 09 & 2.934 & 10 & 4.070 & 7 & 1.094 \\
\hline Segmentados & 76 & 24.776 & 66 & 26.862 & 75 & 11.715 \\
\hline Eosinófilos & 03 & 978 & 09 & 3.663 & 08 & 1.250 \\
\hline Basófilos & 0 & 0 & 0 & 0 & 0 & 0 \\
\hline Linfócitos & 07 & 2.282 & 04 & 1.628 & 09 & 1.406 \\
\hline Monócitos & 05 & 1.630 & 11 & 4.477 & 1 & 157 \\
\hline
\end{tabular}

Legenda: REL - Valores Relativos; ABS - Valores absolutos. 
Após oito dias de internação, o animal começou a apresentar melhora clínica, não apresentava febre e voltou a se alimentar sozinho. Desta forma, foi suspenso o tratamento com dipirona e realizados novos exames para reavaliação. No hemograma foi possível detectar melhora hematológica e ausência de leucocitose (Quadro 1), assim como diminuição dos valores de ALT e AST em relação aos primeiros exames, aumento de FA e diminuição das bilirrubinas (Quadro 2). No sumário de urina, foi detectada diminuição da densidade e melhora da cor, diminuição da albumina, aumento do $\mathrm{pH}$ e diminuição de sangue oculto no exame químico e no exame de sedimento foi detectada presença de hemácias e leucócitos, ausência de cilindros e de cristais de fosfato triplo, comparando-se com o primeiro sumário de urina realizado.

Após dez dias de internação, obteve-se o resultado do exame de cultura de urina para Leptospirose, no qual foi detectada presença de crescimento de leptospiras na cultura para leptospirose e presença de leptospiras na microscopia em campo escuro. Devido à melhora clínica e hematológica do animal e após oito dias de tratamento com ampicilina substituiu-se esta droga por doxiciclina $(5 \mathrm{mg} / \mathrm{kg}, \mathrm{PO}, \mathrm{BID} / 14$ dias $)$. Após 14 dias de internação, o paciente recebeu alta hospitalar com a seguinte prescrição terapêutica domiciliar: doxiciclina $(5 \mathrm{mg} / \mathrm{kg}, \mathrm{PO}, \mathrm{BID} / 10$ dias $)$ e Omeprazol (0,5 mg/kg, PO, SID/20 dias).

Após 38 dias da admissão do animal no Hospital, a proprietária voltou com o mesmo para retorno e relatou que administrou os medicamentos prescritos conforme recomendado e relatou que o animal estava ativo, alimentandose bem, ingerindo água normalmente, com fezes e urina normais. No exame clinico, observou-se mucosas oral (Figura 2), ocular e peniana normocoradas, linfonodos não reativos, TPC $<2$, ativo, frequências cardíaca e respiratória sem alterações e temperatura normal. Em seguida, foram coletados sangue, para avaliação bioquímica da função renal e hepática após o tratamento, e urina para avaliação da leptospiúria por meio do exame de cultura. O resultado do exame bioquímico mostrou normalização dos valores de ALT, AST, FA, bilirrubinas e ureia (Quadro 2). No resultado do exame de cultura de urina para Leptospirose foi possível observar ausência no crescimento de Leptospiras e ausência de leptospiras na microscopia em campo escuro, mostrando ausência de leptospiúria.

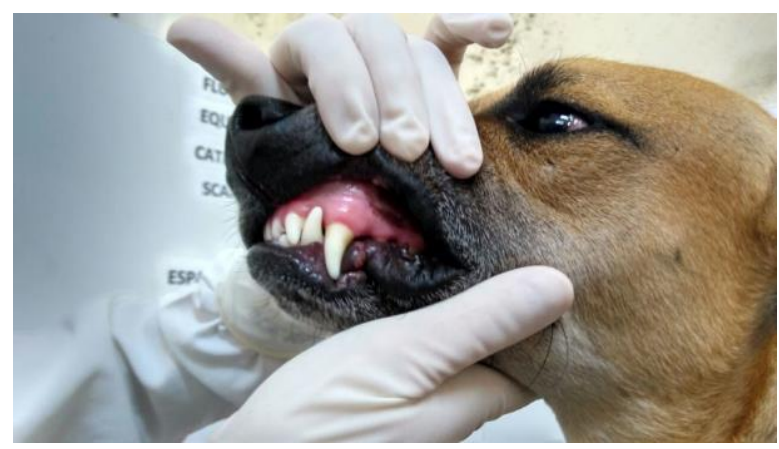

Figura 2. Mucosa oral normocorada, de um cão, após tratamento para leptospirose no HVU/UFPI, Teresina-PI, 2017.

\section{Discussão}

A Leptospirose canina parece ocorrer com mais incidência em cães jovens (Greene et al. 1993; Hagiwara et al. 2015) com idade entre 1-4 anos (Collantes et al. 2016) e em machos (Schuller 2017). Estes dados corroboram com o presente relato em que o paciente tratava-se de um cão macho de um ano e seis meses, apesar de alguns autores não encontrarem relação direta entre idade, sexo, raça e peso com a infecção por leptospiras (Knöpfler et al. 2017).

Quadro 2: Resultado das análises bioquímicas de um cão com leptospirose,nos dias 0, 3, 8 e 38 de tratamento, atendido no HVU/UFPI, Teresina-PI em 2017.

\begin{tabular}{|l|c|c|c|c|}
\hline \multicolumn{1}{|c|}{ Exame } & Dia 0 & Dia 3 & Dia 8 & Dia 38 \\
\hline Uréia (mg/dL) & 76 & 60 & 18,0 & 32,0 \\
Creatinina (mg/dL) & $*$ & 0,3 & 0,4 & 1,0 \\
AST/TGO (U/L) & 253,0 & 228,0 & 41,0 & 25,0 \\
ALT/TGP (U/L) & 108,0 & 231,0 & 102,0 & 20,0 \\
Fosfatase alcalina (U/L) & 148,0 & 293,0 & 394,0 & 42,0 \\
Proteína total(g/dL) & 7,1 & 5,6 & - & - \\
Albumina (g/dL) & 2,5 & 1,4 & - & - \\
Globulina (g/dL) & 4,6 & 4,2 & - & 0,15 \\
Bilirrubina total (mg/dL) & $* *$ & 11,1 & 1,3 & 0,04 \\
Bilirrubina conjugada (mg/dL) & $* *$ & 8,6 & 1,0 & 0,11 \\
Bilirrubina não conjugada (mg/dL) & $* *$ & 2,5 & 0,3 & 3,4 \\
Fósforo (mg/dL) & - & - & 5,3 & \\
\hline
\end{tabular}


Há relatos de aumento da incidência da doença em épocas do ano mais quentes e no período de chuvas (Jorge et al., 2017), assim como relatos de cães infectados que tiveram contato com água contaminada (Hagiwara et al. 2015), acordando com o relato em que o cão acometido parece ter sido infectado devido à água contaminada da chuva que entrou em contato.

Os sorovares de $L$. interrogans que mais sororeagem com amostras de cães são L. australis, $L$. autumnalis, $L$. ballum, $L$. bratislava, $L$. bataviae, L. canicola, L. grippotyphosa, L. hardjo, L. icterohaemorrhagiae, L. pomona e L. tarassovi (Hagiwara et al. 2015). No caso relatado, foi confirmada infecção por Leptospira interrogans, sorovar australis, na titulação de 1:100 pelo MAT.

Os sinais clínicos mais comumente observados nos cães infectados por leptospiras são êmese, letargia e anorexia (Hagiwara et al. 2015; Panigrahi et al. 2015; Knöpfler et al. 2017), seguido de sinais menos observados como febre, icterícia, dor abdominal (Panigrahi et al. 2015) e hematúria (Collantes et al. 2016). Knöpfler et al. (2017) observaram que os sinais mais comuns foram letargia (96\%), anorexia (88\%), êmese (85\%) e dor abdominal (39\%) e apenas $15 \%$ dos animais apresentaram febre e $10 \%$ icterícia. Estes dados se assemelham com os sinais clínicos apresentados pelo animal do relato que foram hiporexia, febre, icterícia e letargia. O sinal de êmese não foi observado, o que pode ter relação com o fato de ser um sinal clínico associado ao acometimento renal (Hagiwara et al. 2015) não visualizado no animal do relato. A icterícia observada em animais com leptospirose ocorre devido à lesão hepatocelular grave e colestase intra-hepática associada (Hagiwara, 2015). Entretanto, Collantes et al. (2016) referem que é um sinal incomum e não confiável para indicar que o cão deve ser testado para a doença, visto que todos os animais ictéricos do seu estudo não tinham leptospirose.

Nas análises hematológicas apresentadas no relato, foi encontrada trombocitopenia que, segundo Schuller (2017), ocorre devido à destruição imuno-mediada, sequestro esplênico ou ativação de plaquetas ou endotélio pelo organismo. Trata-se de um achado hematológico comum em cães com leptospirose (Greene et al. 1993; Hagiwara et al. 2015; Schuller 2017), apesar de $72 \%$ dos cães infectados por leptospira estudados por Collantes et al. (2016) apresentarem plaquetas normais. Leucocitose por neutrofilia também foi detectado no hemograma do cão relatado, corroborando os dados de (Hagiwara et al. (2015); Panigrahi et al. (2015); Knöpfler et al. (2017)) e Schuller (2017). Hagiwara et al. (2015) relata que a ocorrência de leucocitose e febre em animais com leptospirose ocorre devido à síndrome da resposta inflamatória sistêmica associada à sepse. Por outro lado, o sinal de leucopenia foi observado por Greene et al. (1993) Collantes et al. (2016), mas provavelmente tratase de um sinal transitório que ocorre no início da fase aguda da doença, durando 1-2 dias, alterando em seguida para leucocitose. Anemia foi outro achado hematológico importante destacado por Knöpfler et al. (2017) e Schuller (2017), corroborando com o presente relato em que o animal em questão inclusive precisou ser submetido à transfusão de sangue, devido à anemia grave. Nas amostras bioquímicas, o cão do relato apresentou elevação de ALT, AST, FA e bilirrubinas, estando em acordo com os resultados encontrados por (Panigrahi et al. (2015); Knöpfler et al. (2017)) e Schuller (2017). Todavia, estes mesmos autores relataram acometimento renal grave (aumento dos níveis séricos de creatinina e ureia), não observada de forma significativa no presente relato que mostrou apenas um aumento leve de ureia e valores normais de creatinina. Em relação ao sumário de urina, os resultados apresentados no relato condizem com Knöpfler et al. (2017) que destaca hematúria e presença de células epiteliais, leucócitos, cilindros e cristais na análise do sedimento. Todavia, este mesmo autor e Schuller (2017) destacam também a ocorrência de glicosúria, não visualizada no sumário de urina do cão relatado. O tratamento da Leptospirose canina é por meio de antibioticoterapia, sendo a Doxiciclina o antibiótico de eleição, como especificado pelo Grupo de Estudos sobre Leptospirose da American College of Veterinary Internal Medicine no Consensus Statement on Leptospirosis (Hagiwara et al. 2015). Todavia, animais com êmese, acometimento renal ou hepático ou em anorexia, não toleram a Doxiciclina, sendo indicada terapia inicial com Penicilina ou derivados e terapia de suporte (Hagiwara (Hagiwara et al. 2015; Knöpfler et al. 2017; Schuller 2017). No presente relato, o cão foi tratado inicialmente com Penicilina Benzantina, antes de confirmar o diagnóstico de Leptospirose, corroborando com Hagiwara et al. (2015) que indica iniciar o tratamento o quanto antes possível ao suspeitar de leptospirose, antes mesmo da obtenção dos resultados de exames confirmatórios. Com o diagnóstico confirmado, o 
antibiótico foi substituído para Ampicilina por via intravenosa até o animal voltar a se alimentar sozinho e os valores das enzimas hepáticas diminuírem, após o que finalmente foi instituída terapia com $5 \mathrm{mg} / \mathrm{kg}$ de Doxiciclina por via oral durante 14 dias, para eliminar a leptospira dos túbulos renais. Schuller (2017) relatou que a Doxiciclina foi capaz de eliminar a leptospiúria e as leptospiras dos rins de ratos.

Alguns autores relatam que após a infecção por leptospiras, o animal pode apresentar sequelas como hepatite crônica, como relatado em cães infectados pelo sorovar Australis (Schuller 2017) e sorogrupo Australis (Hagiwara et al. 2015). No entanto, este dado não foi visualizado no cão do relato que, após o tratamento, demonstrou valores normais das enzimas hepáticas no exame bioquímico com 38 dias desde o dia da admissão no Hospital Veterinário.

\section{Conclusão}

A Leptospirose canina, quando diagnosticada na forma inicial e dado início ao tratamento adequado no momento em que surge a suspeita do diagnóstico, as chances de sucesso no tratamento são maiores. Além disso, por se tratar de uma zoonose, deve-se ter em mente que não apenas o animal acometido está em risco, como também seus proprietários e toda a equipe que entrará em contato com o animal durante o tratamento, sendo de extrema importância medidas sanitárias de proteção.

\section{Referências Bibliográficas}

Collantes T.M.A., David J.M.F., Vergara E.J.S., Armea S.R.D. \& Flores M.L.S. 2016. Detection of pathogenic leptospires and analysis of factors and clinical signs associated with canine leptospirosis. Philippine Journal of Veterinary and Animal Sciences 42, 41-89.

Greene C.E., Samperio J.O. \& Gómez J.P. 1993. Enfermedades infecciosas: Perros y gatos. Editora Interamericana, São Paulo.
Hagiwara M.K., Miotto B.A. \& Kogika M.M. 2015. Leptospirose. In: Tratado de medicina interna de cães e gatos (eds. by Jericó MM, Neto JPA \& Kogika MM), pp. 2678-708. Roca, Rio de Janeiro, Brasil.

Jorge, S., Schuch, R.A., Oliveira, N. R., Cunha, C.E.P., Gomes, C.K., Oliveira, T.L., Rizzi, C., Qadan, A.F., Pacce, V.D., Recuero, A.L.C., Brod, C.S. \& Dellagostin, O.A. 2017. Human and animal leptospirosis in Southern Brazil: a five-year retrospective study. Travel Medicine and Infectious Disease, 18, 46-52.

Knöpfler S., Mayer-Scholl A., Luge E., Klopfleisch R., Gruber A.D., Nöckler K. \& Kohn B. 2017. Evaluation of clinical, laboratory, imaging findings and outcome in 99 dogs with leptospirosis. Journal of Small Animal Practice 58, 582-8.

Nelson R.W. \& Couto C.G. 2015. Medicina interna de pequenos animais. Elsevier Editora, Amsterdan.

Panigrahi P.N., Behera S.K. \& Dey S. 2015. Diagnosis and clinical management of leptospirosis in a dog. Intas Polivet 16, 361-4.

Schuller S. 2017. Leptospirosis. In: Textbook of veterinary internal medicine: diseases of the dog and the cat (eds. by Ettinger D \& Feldman E), pp. 2335-43. Elsevier, Missouri, USA.

Schuller S., Francey T., Hartmann K., Hugonnard M., Kohn B., Nally J. \& Sykes J. 2015. European consensus statement on leptospirosis in $\operatorname{dogs}$ and cats. Journal of Small Animal Practice 56, 159-79.

Article History:

Received 4 April 2018

Accepted 26 April 2018

Available online 31 May 2018

License information: This is an open-access article distributed under the terms of the Creative Commons Attribution License 4.0, which permits unrestricted use, distribution, and reproduction in any medium, provided the original work is properly cited 